\title{
Real-Space Imaging of Atomic Structure with White X Rays
}

\author{
P. Korecki and G. Materlik \\ Hamburger Synchrotronstrahlungslabor HASYLAB am Deutschen Elektronen-Synchrotron DESY, 22603 Hamburg, Germany
}

(Received 9 August 2000)

\begin{abstract}
The first real-space x-ray image of an atomic structure was obtained by illuminating a crystal with white synchrotron radiation. The internal photocurrent signal served as a probe of the x-ray interference field strength at the atomic sites and was accordingly measured as a function of illumination direction to record the two-dimensional image. This novel method of real-space imaging makes use of the fact that the interference field intensity is energy independent with respect to contributions from those scattering atoms which are brought via sample rotation into the forward scattering condition. In contrast, contributions from other atoms oscillate with energy and vanish for broadband illumination.
\end{abstract}

DOI: 10.1103/PhysRevLett.86.2333

Although $\mathrm{x}$ rays have a well suited wavelength for realspace atomic structure imaging, the experimental resolution has so far never reached the wavelength limit. The small numerical apertures of $\mathrm{x}$-ray lenses limit the resolution to at best $10 \mathrm{~nm}$, and thus the atomic resolution $\mathrm{x}$-ray microscope is not feasible [1]. A lensless approach such as a point-projection microscope [2] is limited by the finite size of the $\mathrm{x}$-ray source, which in order to accomplish high magnification has to be brought close to the object. X-ray diffraction methods, on the other hand, can be used only for crystals and, in addition, the so called phase problem makes the direct inversion of the diffracted intensities from reciprocal to real space impossible.

Internal source holography (ISH) [3] and internal detector holography (IDH) [4] are alternative lensless approaches which were realized experimentally quite recently. They avoid optical elements and thus provide images with atomic resolution by using $\mathrm{x}$ rays [5] or $\gamma$ rays [6]. In ISH, atoms inside a sample serve as coherent point sources of fluorescent radiation which is scattered from nearby atoms. This produces an interference pattern corresponding to the local environment around the emitting atoms. Since $\mathrm{x}$-ray fluorescence radiation has a sufficient temporal coherence length $\left(l_{c} \simeq 1 \mu \mathrm{m}\right)$ to image atomic distances, such a pattern is an in-line Gabor hologram [7]. The reconstruction of the hologram to real space is performed numerically [8]. In IDH, the interference of elastically scattered waves is monitored by measuring the absorption of particular atoms serving as internal detectors. The detection signal for measuring the local $\mathrm{x}$-ray field intensity is provided by $\mathrm{x}$-ray fluorescence or electron yield and is recorded with an external detector. Because of the weak scattering of $\mathrm{x}$ rays the holographic modulation is only in the order of $10^{-4}$ of the total signal. In addition, for high spatial resolution, holograms have to be recorded over wide angular and energy ranges. Therefore, high-brilliance sources and fast two-dimensional energy resolving detectors are needed to make these techniques widely applicable.

In this Letter we demonstrate that the atomic structure can be viewed directly in real space by using incoherent white $\mathrm{x}$ rays in connection with atoms acting as internal
PACS numbers: 61.10. $-\mathrm{i}, 07.85 . \mathrm{Tt}, 42.40 .-\mathrm{i}, 61.14 .-\mathrm{x}$

detectors. We show the first projection phase-contrast image of an atomic structure recorded with $\mathrm{x}$ rays.

When the sample is illuminated by polychromatic radiation, described by the wave number spectrum $N(k)$ and the illumination direction $\hat{\mathbf{k}}$, the $\mathrm{x}$-ray field intensity at a given atomic detector can be evaluated as an incoherent superposition of wave fields each corresponding to a given $k$ vector value, i.e., as $I(\hat{\mathbf{k}})=\int_{0}^{\infty} N(k)|E(\mathbf{k})|^{2} d k$. In the first Born approximation, $E(\mathbf{k})$ may be written as a sum of the incident plane wave $E_{0}(\mathbf{k})$ and the superposition $E_{s}(\mathbf{k})$ of all singly scattered waves:

$$
\begin{aligned}
I(\hat{\mathbf{k}}) & \propto \int_{0}^{\infty} N(k)\left|E_{0}(\mathbf{k})+E_{s}(\mathbf{k})\right|^{2} d k \\
& =\int_{0}^{\infty} N(k)\left[\left|E_{0}(\mathbf{k})\right|^{2}+2 \operatorname{Re} E_{0}^{*}(\mathbf{k}) E_{s}(\mathbf{k})+\ldots\right] d k .
\end{aligned}
$$

The factor $\left|E_{0}(\mathbf{k})\right|^{2}$ provides the isotropic background whereas the terms containing the squared scattered waves can be neglected in a weak scattering approximation. The background normalized signal at the atomic detector sites is $\chi(\hat{\mathbf{k}})=\left[I(\hat{\mathbf{k}})-I_{0}(\hat{\mathbf{k}})\right] / I_{0}(\hat{\mathbf{k}})$. It is related to the second term on the right side of Eq. (1) and can be written as [9]

$$
\chi(\hat{\mathbf{k}}) \propto-r_{0} \operatorname{Re} \int_{0}^{\infty} d k N(k) \int_{V} \frac{1}{r} \rho(\mathbf{r}) e^{i(k r+\mathbf{k r})} d V,
$$

where $\rho(\mathbf{r})$ is the electron density at position $\mathbf{r}$ relative to the absorbing atom, $r_{0}$ is the classical electron radius, and the minus sign reflects the phase change of the scattered waves. Equation (2) neglects the attenuation of the scattered waves. For monochromatic illumination, i.e., $N(k)=$ $\delta\left(k-k_{0}\right)$, Eq. (2) becomes the formula used in ISH.

Consider, however, the case of perfect white illumination of the sample. For $N(k)=1$ the integration yields

$$
\chi(\hat{\mathbf{k}}) \propto-r_{0} \int_{V} \frac{1}{r} \rho(\mathbf{r}) \delta(r+\hat{\mathbf{k}} \mathbf{r}) d V .
$$

The presence of the Dirac function with argument $r+$ $\hat{\mathbf{k}} \mathbf{r}$ means that, for a given illumination direction, only the 
atoms along the line $r=-\hat{\mathbf{k}} \mathbf{r}$ (i.e., along the illumination direction) contribute to the signal modulations. Therefore, when the sample is illuminated by a white beam a real-space spherical projection of the electron density is obtained, since all diffraction patterns corresponding to different energies add up constructively only in the forward direction, where the scattered waves are all in phase. The $1 / r$ factor in Eq. (3) enhances the contribution from the nearest scatterers. $\chi(\hat{\mathbf{k}})$ will thus have minimum values for directions corresponding to the nearest neighbors, and hence the information about bond directions can be obtained directly without performing any integral transform. This direct real-space imaging works only when all detecting atoms have similar neighborhoods. Then the scattering geometry can be viewed as the time-reversed version of a lensless projection $\mathrm{x}$-ray microscope [10] with an internal atomic source of illumination.

However, because of experimental limitations, it is necessary to consider the intermediate case of a band-limited $N(k)$ spectrum approximating the incident radiation spectrum by a Gaussian curve centered at $k_{0}$ and spread by $\Delta k$. Figure 1 shows diffraction patterns $\left(k_{0}=20 \AA^{-1}\right)$ of a point scatterer probed by an atomic detector at a distance of $r=2.35 \AA$ calculated for several values of $\Delta k$. As the spectrum of incident radiation becomes broader, the diffracted wavelets localize around the forward scattering direction, and simultaneously their modulation amplitude is damped. In other words, the higher-order interference fringes vanish and only the zeroth-order diffraction dip survives in the forward direction. The increase of $\Delta k$ reduces the temporal coherence length $l_{c}=\lambda^{2} / \Delta \lambda=2 \pi / \Delta k$, while the transverse coherence is preserved. For practical purposes, this means that by using a broadband spectrum $\left(k_{0} / 2<\Delta k<k_{0} / 5\right)$, one is also able to determine the directions of the nearest bonds, without any a priori knowledge because the scattering contribution from a single atom is localized around the corresponding interatomic

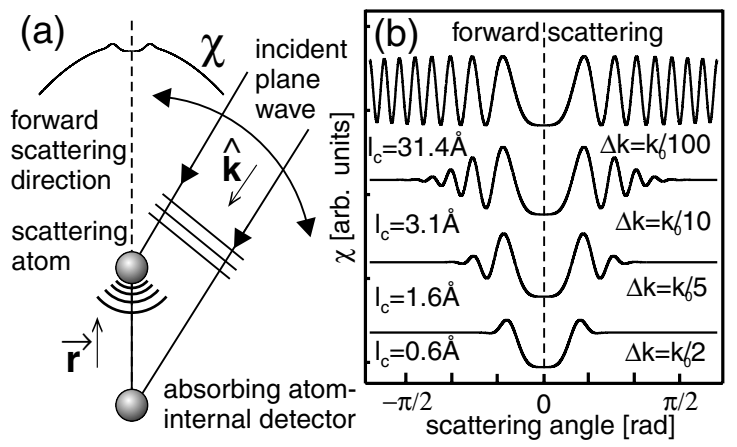

FIG. 1. Diffraction of a plane wave $\left(k_{0}=20 \AA^{-1}\right)$ by a single point scatterer probed by an atomic detector at a distance of $r=2.35 \AA$. (a) Scattering geometry. (b) Diffraction patterns calculated for several $\Delta k$ values. Note the localization of the diffraction pattern around the interatomic direction as the wavelength spread is increased. direction. Therefore, Fig. 1 shows a transition from coherent imaging of a structure in reciprocal space to incoherent imaging in real space. Figure 1 also demonstrates that, even for relatively broadband illumination $\left(\Delta k \simeq k_{0} / 10\right)$, a holographic approach may be applied to image the nearest atoms as was done using Bremsstrahlung photons in Ref. [11].

An incoherent image in real space was recorded at the Hamburg Synchrotron Radiation Laboratory HASYLAB/ DESY using the beam line D4. The scheme of the experimental setup is shown in Fig. 2(a). The direct white radiation from the bending magnet passed through a $7.6 \mathrm{~mm}$ thick aluminum absorber that was used to shape a broadband energy spectrum. A Si photodiode served as a test sample. Photodiodes are efficient detectors not only for visible light but also for hard $\mathrm{x}$ rays [12]. $\mathrm{X}$ rays produce high-energy photoelectrons and Compton electrons which are "thermalized" into a distribution of electron-hole pairs with energies around $3.6 \mathrm{eV}$ for Si. Since the Si photodiode is a perfect crystal, it is well suited to serve as sample and detector simultaneously. The photocurrent of the diode measures simply the total absorption yield. The diode was an ion implanted $300 \mu \mathrm{m}$ thick $n$-type wafer with a miscut of $7^{\circ}$ relative to the (111) surface [13]. The diode was operated without bias in the photovoltaic mode as a current source and the photocurrent was amplified by typically $10^{6} \mathrm{~V} / \mathrm{A}$. In the experiment, the diode was rotated around two axes. The inclination angle $\theta$, measured with respect to the surface normal, was changed in $1^{\circ}$ steps. The $\phi$ rotation around the surface normal was performed continuously and the measured signal was integrated in $0.5^{\circ}$ intervals. The measuring time of a single pattern $\left(0^{\circ}<\theta<55^{\circ}, 0^{\circ}<\phi<360^{\circ}\right)$ was three hours. Eight patterns were added together. About $10^{9}$ photons/s/pixel were absorbed by the sample. The measured signal was normalized using a similar photodiode placed directly in front of the sample.

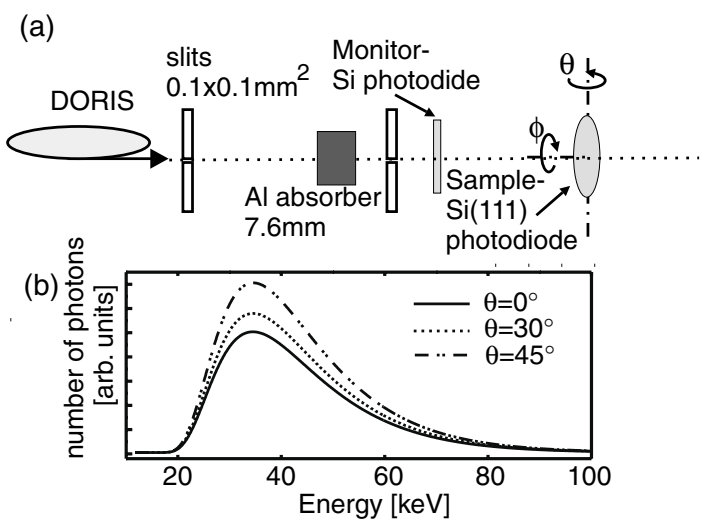

FIG. 2. White beam experiment: measurement of the total absorption yield as a function of sample illumination direction. (a) Experimental setup. (b) Calculated energy spectrum seen by the average atomic detector inside the sample diode shown for various inclination angles. 
An average absorbing sample atom sees a wave-number spectrum that depends first on the spectrum of the beam produced at the bending magnet, second, on the absorption and scattering of all elements placed into the beam, and finally on the integrated absorption in the sample. The function $N(k)$ also contains the different energy-current conversion ratios for photoelectrons and Compton electrons. The energy spectrum $N(\epsilon)$, with $\epsilon=\hbar c k$, calculated for an aluminum absorber with a thickness of $7.6 \mathrm{~mm}$ is presented in Fig. 2(b) for several inclination angles. The spectrum has its maximum around $35 \mathrm{keV}$ with FWHM of ca. $25 \mathrm{keV}$. Its shape does not change essentially with the inclination angle. The calculated $N(\epsilon)$ was checked by comparison with the similar spectrum measured with a crystal monochromator. The longitudinal coherence length of such a beam is less than $1 \AA$.

Figure 3(a) shows the recorded pattern of the total absorption measured as a function of sample orientation relative to the $\mathrm{x}$-ray beam direction. A simple, slowly varying two-dimensional background subtraction and a threefold symmetry operation were applied to the data. The relative signal modulation is of the order of $4 \times 10^{-4}$. Note that the modulation has the same order of magnitude as the forward scattering amplitude on a nearest neighbor $\mathrm{Si}$ atom: $f_{\mathrm{Si}} / r=Z r_{0} / r=1.6 \times 10^{-4}$. This shows that the largest contributions to the pattern come from the local structure.

Figure 3(b) shows the absorption pattern simulated for experimental conditions according to Eq. (2) and with the energy spectrum shown in Fig. 2(b). The calculation was done for two nonequivalent crystal sites: $\mathrm{Si}(1)$ in position 000 and $\mathrm{Si}(2)$ in position $\frac{1}{4} \frac{1}{4} \frac{1}{4}$ of the unit cell within a $120 \AA$ diameter cluster. The agreement with the measured pattern is very good. The reliability factor has a value of $R=0.075$ [14]. Some discrepancies in the fine structures can result from the finite size of the cluster used in

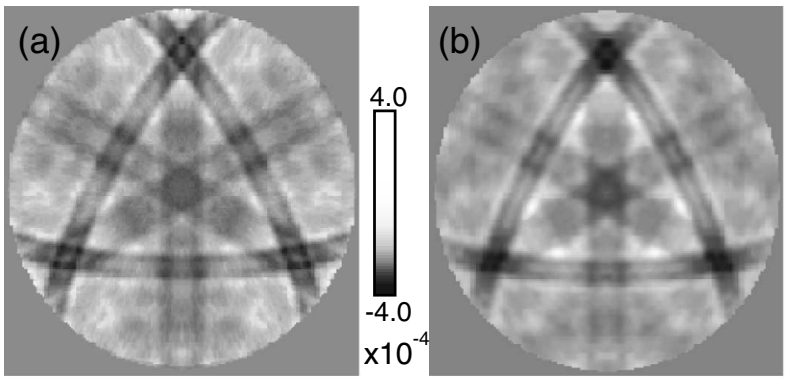

FIG. 3. (a) The experimental pattern of total absorption yield caused by white illumination of the $\mathrm{Si}(111)$ crystal measured as the function of sample orientation. The linear gray scale is proportional to the sample current (dark: low absorption; bright: high absorption). The observed modulation of the total signal is about $4 \times 10^{-4}$. (b) Pattern simulated for an atomic cluster with radius of $60 \AA$ around two nonequivalent atomic sites in $\mathrm{Si}$. Both patterns are shown in spherical projection. The center corresponds to the $\langle 111\rangle$ direction and the edge to $48^{\circ}$ away from it. the calculation and from multiple-scattering effects, which were not taken into account. The single-scattering approximation can be applied because the broadband illumination reduces the effective number of scatterers from $N^{3}$ atoms in a crystal volume to $N$ atoms in each atomic row along the illumination direction. In addition, for $x$ rays the scattering amplitude of a single atom has a very small value and thus the multiple-scattering contribution is very weak.

Apart from the inverse contrast resulting from the negative value of the photon scattering amplitude, the measured pattern is very similar to photoelectron forward focusing patterns measured for the $\mathrm{Si}(111)$ surface [15]. Forward scattering patterns of low-energy electrons [16] and channeling patterns [17] of high-energy electrons are routinely used for simple and direct real-space determination of bond direction at surfaces, at interfaces, and in bulk crystals. Despite the different scattering mechanism, the x-ray pattern may be analyzed in the same way to provide structural information in real space [18].

To demonstrate the sensitivity of the measured pattern to structural parameters we performed calculations separately for atoms in clusters around the two nonequivalent atomic sites in Si. Patterns calculated for detecting atoms in positions $\mathrm{Si}(1)$ and $\mathrm{Si}(2)$ are shown in Figs. 4(a) and 4(b), respectively. Those patterns are less similar to the
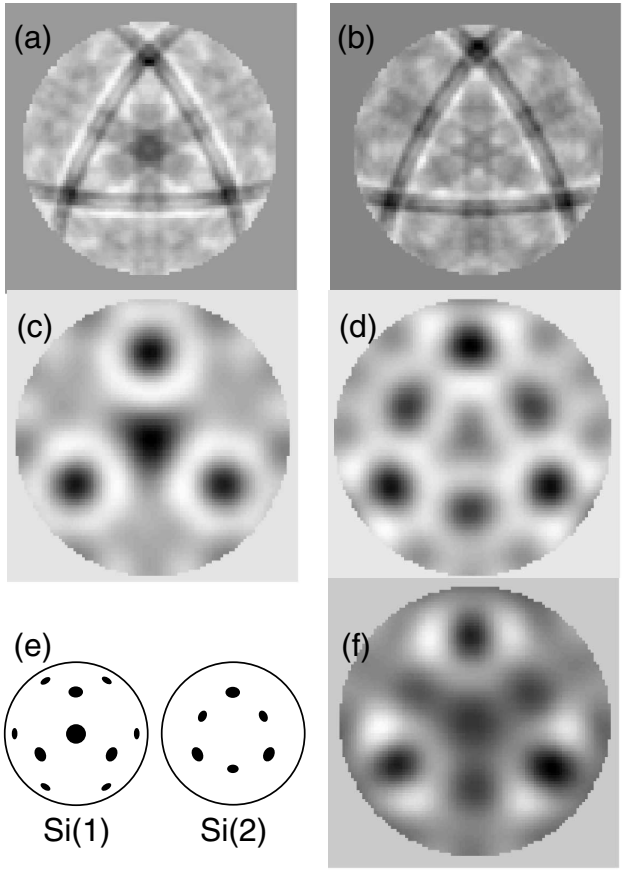

FIG. 4. (a),(b) Patterns of total absorption simulated separately for clusters around atomic detectors in the two nonequivalent positions $\mathrm{Si}(1)$ and $\mathrm{Si}(2)$ in the unit cell. (c),(d) Low-pass filtered patterns from (a) and (b). (e) Geometrical projection of atoms in the $5 \AA$ neighborhood of $\mathrm{Si}(1)$ and $\mathrm{Si}(2)$ sites. (f) Low-pass filtered image of the experimental pattern from Fig. 3(a) showing superimposed images of atoms above $\mathrm{Si}(1)$ and $\mathrm{Si}(2)$ atomic sites. 
experimental data than is their superposition. The corresponding $R$ factors are 0.12 and 0.17 which means nearly $100 \%$ change with respect to the summed pattern. This indicates the possibility of conventional $R$ factor analysis based on simple kinematical calculations.

Because of the weak absorption of $\mathrm{x}$ rays, the measured pattern contains features coming from distant atoms. This contribution could cover the signal from the nearest atoms. To demonstrate that real-space local information can be extracted, a low-pass filter was applied to the calculated data. The low-pass filter is not ideally suited for this purpose since the signal from an individual atom is neither distributed over the whole hemisphere nor is it a simple sine wave. Nevertheless, this procedure provides the angular positions of nearest scatterers as shown in Fig. 4. Figures 4(c) and 4(d) show data calculated for $\mathrm{Si}(1)$ and $\mathrm{Si}(2)$ atomic detectors after application of a low-pass filter. The geometrical projections of nearest atoms (up to $5 \AA$ ) around the $\mathrm{Si}(1)$ and $\mathrm{Si}(2)$ sites are drawn in Fig. 4(e). The minimum values of the low-pass filtered images correspond exactly to the geometrical projections of the atoms in Fig. 4(e). The low-pass filtered version of the experimental pattern in Fig. 4(f) shows a superposition of the atomic images around $\mathrm{Si}(1)$ and $\mathrm{Si}(2)$ sites. The suppressed higherorder interference fringes which contain the information about interatomic distances are distorted by the Fourier filter. However, the formalism described in Ref. [18] or the wavelet approach [19] can be applied to give the full three-dimensional local structure.

What are the practical applications of the ideas presented in this paper? The signal is weak; however, the huge intensity of "white" synchrotron radiation beams allows it to be measured in a simple manner. Moreover, the scattering is stronger in the forward direction than in any other direction. It should be possible to detect a characteristic radiation coming from fluorescence or Auger processes, instead of the total absorption yield. By this means, element selectivity will be achieved, and the local structure of dopant atoms or atoms in buried layers could be visualized in a manner not accessible by standard methods and supplementary to the extended x-ray absorption fine structure method.

As a simple example, consider a tetragonal distortion of a bcc unit cell. A distortion of $5 \%$ will cause a $3^{\circ}$ angular shift of atomic images. In contrast to x-ray holography, this information is obtained in real space. This means that the signal does not need to be measured on the whole hemisphere. If only particular directions are of interest the data can be measured as one-dimensional angular scans. By changing the energy spectrum it will be possible to move from space to frequency localization and to achieve more high-resolution information about bond lengths.

We thank Dr. Dimitri Novikov for help during the experiment and Dr. Yoshinori Nishino for valuable discussions.

[1] C. Jacobsen, J. Microsc. 197, 173 (2000).

[2] I. McNulty, Nucl. Instrum. Methods Phys. Res., Sect. A 347, 170 (1994).

[3] A. Szöke, in Short Wavelength Coherent Radiation: Generation and Application, edited by D. T. Attwood and J. Bokor, AIP Conf. Proc. No. 147 (AIP, New York, 1986).

[4] T. Gog et al., Phys. Rev. Lett. 76, 3132 (1996).

[5] M. Tegze and G. Faigel, Nature (London) 380, 49 (1996); M. Tegze et al., Nature (London) 407, 38 (2000); T. Hiort et al., Phys. Rev. B 61, R830 (2000).

[6] P. Korecki , J. Korecki, and T. Slezak, Phys. Rev. Lett 79, 3518 (1997).

[7] D. Gabor, Nature (London) 161, 777 (1948).

[8] J. J. Barton, Phys. Rev. Lett. 61, 1356 (1988).

[9] P. M. Len et al., Phys. Rev. B 55, R3323 (1997); B. Adams et al., Phys. Rev. B 57, 7526 (1998).

[10] S. Lagomarsino et al., Appl. Phys. Lett. 71, 2557 (1997).

[11] S. G. Bompadre, T. W. Petersen, L. B. Sorensen, Phys. Rev. Lett. 83, 2741 (1999).

[12] J. P. Kirkland et al., Nucl. Instrum. Methods Phys. Res., Sect. A 266, 602 (1988).

[13] The IP 300-300-PH Eurisys Si detector was used in the experiment.

[14] We used an $R$ factor defined as $R=\sum\left(I_{\text {calc }}-I_{\text {exp }}\right)^{2} /$ $\sum\left(I_{\text {calc }}+I_{\text {exp }}\right)^{2}$. The value below 0.1 shows good agreement between calculated and experimental 2D data.

[15] J. Osterwalder et al., J. Electron Spectrosc. Relat. Phenom. 68, 1 (1994); C. Westphal et al., Surf. Sci. 462, 103 (2000).

[16] S. A. Chambers, Surf. Sci. Rep. 16, 261 (1992); S. Mróz, Surf. Rev. Lett. 4, 117 (1997).

[17] J. C. H. Spence and J. Tafto, J. Micros. 130, 147 (1983).

[18] M. Seelmann-Eggebert, Phys. Rev. B 55, 15842 (1997).

[19] Wavelets: A Tutorial in Theory and Applications, edited by C. K. Chui (Academic, New York, 1992), and references therein. 